INPLASY

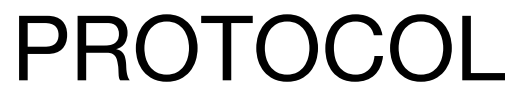

To cite: Li et al.

Clinicopathological

significance of Ki67 expression

in colorectal cancer: a protocol

of systematic review and

meta-analysis. Inplasy protocol

202030009. doi:

10.37766/inplasy2020.3.0009

Received: 27 March 2020

Published: 28 March 2020

Corresponding author: Jing Li

JingLi198001@outlook.com

Author Affiliation:

Jiamusi University School of

Basic Medical Science

Support: SRPHLJPHHC

(2019-326)

Review Stage at time of this submission: The review has not yet started.

\section{Clinicopathological significance of Ki67 expression in colorectal cancer: a protocol of systematic review and meta-analysis}

Li J1; Liu, Z2; Yu, H³ Xue, Q4; He, W5; Yu, H6.

\begin{abstract}
Review question: Is Ki67 expression accurate to diagnose colorectal cancer(CC)?

Methods: Electronic searches A comprehensive search for associated articles in electronic bibliographic databases (MEDLINE, EMBASE, Cochrane Library, Web of Science, Chinese Biomedical Literature Database, and China National Knowledge Infrastructure) will be carried out from inception to the February 29, 2020. There will be no language and publication status restrictions. We will build search strategy sample for MEDLINIE, and we will adapt similar search strategies for other databases. Other resources We will check and obtain potential studies from clinical trial registry, conference abstracts, and reference lists of relevant reviews..
\end{abstract}

INPLASY registration number: This protocol was registered with the International Platform of Registered Systematic Review and Meta-Analysis Protocols (INPLASY) on 27 March 2020 and was last updated on 27 March 2020 (registration number INPLASY202030009.

Conflicts of interest:

None.

\section{INTRODUCTION}

Objectives / Review question: Is Ki67 expression accurate to diagnose colorectal cancer(CC)?

Condition being studied: Colorectal cancer and Ki67 expression.

\section{METHODS}

Participant or population: We will include case-controlled studies (CCSs) that compare Ki67 expression between CC participants with $\mathrm{CC}$ tissues and normal adjacent tissues. We will not employ any restrictions to the age, sex, race, and CC severity. 
Intervention: Index test: We will include studies that specify the index test utilized for the measurement of Ki67 expression to diagnose potential patients with $\mathrm{CC}$.

Comparator: Reference test: Any patients with histological-proven $\mathrm{CC}$ will be included.

Study designs to be included: All CCSs that explored the diagnostic accuracy of Ki67 expression in CC will be included. We will not apply restrictions to the basis of language of $p$.

Eligibility criteria: We will include CCSs that compare Ki67 expression with histologicalproven $\mathrm{CC}$ between $\mathrm{CC}$ tissues and normal adjacent tissues in $\mathrm{CC}$ patients. We will not employ any restrictions to the age, sex, race, and CC severity.

Information sources: Electronic searches A comprehensive search for associated articles in electronic bibliographic databases (MEDLINE, EMBASE, Cochrane Library, Web of Science, Chinese Biomedical Literature Database, and China National Knowledge Infrastructure) will be carried out from inception to the February 29, 2020. There will be no language and publication status restrictions. We will build search strategy sample for MEDLINIE, and we will adapt similar search strategies for other databases. Other resources We will check and obtain potential studies from clinical trial registry, conference abstracts, and reference lists of relevant reviews.

Main outcome(s): Primary outcomes are sensitivity and specificity. Secondary outcomes are positive likelihood ratio, negative likelihood ratio, and diagnostic odds ratio.

Data management: After study selection, two authors will independently collect information from all eligible studies. Any differences will be solved by a third author with consultation. We will extract following information: first author, year of publication, country, sample size, age, sex, CC severity, Ki67 expression test, reference test, outcomes, results, conclusions, and conflict of interest.

Quality assessment / Risk of bias analysis: Two authors will independently appraise methodological quality using Quality Assessment of Diagnostic Accuracy Studies tool. We will assess it through four aspects. Any different conflicts will be settled by a third experienced author through consultation.

Strategy of data synthesis: RevMan V.5.3 software and Stata V.12.0 software will be employed to carry out statistical analysis. We will estimate outcome values as descriptive statistics and $95 \%$ confidence intervals. $\left.\right|^{2}$ statistic will be utilized to check heterogeneity across eligible studies. $1^{2} \leq$ $50 \%$ implies homogeneity, and we will use a fixed-effects model. On the other hand, I $^{2}$ $>50 \%$ reveals discrete heterogeneity, and we will exert a random-effects model. We will estimate values of outcome data (e.g. sensitivity, specificity) using $2 \times 2$ tables. Additionally, we will estimate a descriptive forest plot and a summary receiver operating characteristic plot. If there is homogeneity among included studies, we will perform a meta-analysis based on the similarity in characteristics of study and patient, and outcomes. If there is obvious heterogeneity, we will scrutinize its sources using a subgroup analysis and bivariate random-effects regression approach.

Subgroup analysis: Whenever necessary, we will investigate sources of apparent heterogeneity based on the differences in study characteristics, study quality and outcomes.

Sensibility analysis: Whenever necessary, we will examine the stability of study results by eliminating low quality studies.

\section{Countries involved: China.}

Keywords: Colorectal cancer; Ki67 expression; accuracy; sensitivity; specificity. 